Voix et Images

volxetimages

\title{
Bibliographie de Jacques Poulin
}

\section{Jean-Pierre Lapointe et Jean Levasseur}

Volume 15, numéro 1 (43), automne 1989

Jacques Poulin

URI : https://id.erudit.org/iderudit/200816ar

DOI : https://doi.org/10.7202/200816ar

Aller au sommaire du numéro

Éditeur(s)

Université du Québec à Montréal

ISSN

0318-9201 (imprimé)

1705-933X (numérique)

Découvrir la revue

Citer ce document

Lapointe, J.-P. \& Levasseur, J. (1989). Bibliographie de Jacques Poulin. Voix et Images, 15(1), 58-64. https://doi.org/10.7202/200816ar d'utilisation que vous pouvez consulter en ligne.

https://apropos.erudit.org/fr/usagers/politique-dutilisation/ 


\section{Bibliographie de Jacques Poulin}

\section{par Jean-Pierre Lapointe, université Trent et Jean Levasseur, université Mount Saint Vincent}

\section{CEuvres de Jacques Poulin}

Mon cheval pour un royaume, Montréal, Éditions du Jour, 1967; Montréal, Leméac, 1987 (Poche Québec littérature).

Jimmy, Montréal, Éditions du Jour, 1969; Montréal, Leméac, 1978; Montréal, Stanké, 1985 (Québec 10/10).

Le Cour de la baleine bleue, Montréal, Éditions du Jour, 1970; Montréal, Éditions du Jour, 1979 (le Petit Jour); Montréal, Leméac, 1987.

The «Jimmy» Trilogy, traduction de Sheila Fischman, Toronto, Anansi, 1979.

Faites de beaux rêves, Montréal, l'Actuelle, 1974; Montréal, Bibliothèque québécoise, 1988.

Les Grandes Marées, Montréal, Leméac, 1978; Montréal, Leméac, 1986 (Poche Québec littérature).

Spring Tides, Toronto, Anansi, 1986.

Volkswagen Blues, Montréal, Québec/Amérique, 1984 (Littérature d'Amérique); Montréal, Québec/Amérique, 1987 (Espace).

Volkswagen Blues, Toronto, McLelland and Stewart, 1988.

II. Articles de critique

[ANONYME], «Faites de beaux rêves», le Livre canadien, vol. V, n० 320, 1974.

[ANONYME], «Le Cour de la baleine bleue», le Livre canadien, vol. II, no 85, 1971.

[ANONYME], «Le Caur de la baleine bleue», l'Église canadienne, décembre 1972, p. 308.

[ANONYME], «Plaisir à Jacques Poulin», Ici Radio-Canada, no 339, 8 novembre 1978, [n.p.].

[ANONYME], «Les Prix du Gouverneur général 1979: roman», Lettres québécoises, avril-mai 1979, p. 52.

[ANONYME], «The Jimmy Trilogy», Choice, vol. XVII, mai 1980, p. 395.

ABLEY, Mark, «Seeking the Keys to the Mysteries», Maclean's, 20 août 1979, p. 38-39.

—, «Trouble in Paradise: Review of Spring Tides», Maclean's, 10r septembre 1986, p. 56.

ACKERMAN, Marianne, «The French Connection: Review of Volkswagen Blues», Maclean's, 16 mai 1988, p. 58.

ADACHI, Ken, «Quebec Fiction: A Lively Book and a Bleak One: Review of Spring Tides and Standing Flight», Toronto Star, 10 août 1986, p. A-19.

-, «Poulin's Road Trip is a Kerouac-Like Odyssey», Sunday Star, 8 mai 1988, p. A-22.

ANDREWS, Audrey, «Tender Fable Reveals Man's Loneliness: Review of Spring Tides», Calgary Herald, 21 septembre 1986, p. C8. 
BEAULIEU, Ivanhoé, «Roman québécois. Jacques Poulin. Sans craindre le rejet», le Soleil, 30 janvier 1971, p. 46.

—, «Le deuxième numéro de Nord et Jacques Poulin», le Soleil, 17 mars 1972, p. 58.

—, «Petit collage (intime et fictif...) au sujet d'un écrivain de Québec-la-ville et où il est question de Guy Lafleur», Nord, n² 2, 1972, p. 71-72.

BEAULIEU, Nicole, «L'écrivain dans l'ombre», l'Actualité, vol. X, no 4, avril 1985, p. 73-77.

BEAULIEU, Victor-Lévy, «De la fiction à l'affliction», Digeste éclair, vol. VI, no 4, avril 1969, p. 17.

BELLEMARE, Madeleine, «Le livre du mois: Volkswagen Blues (entrevue)», Nos livres, vol. XV, juin-juillet 1984, p. 4-5.

BILLY, Hélène de, «Une Amérique panoramique sur la pointe des pieds», le Devoir, 19 mai 1984 , p. $25,33$.

BILODEAU, Line, «Mon cheval pour un royaume», Dictionnaire des cuuves littéraires du Québec, tome IV, 1960-1969, Montréal, Fides, 1984, p. 585-587.

BOIVIN, Aurélien, «Bibliographie», Québec français, mai 1979, p. 40 (reproduit dans Romanciers du Québec, Québec, Québec-français, 1980, p. 160-161).

BONIN, Raymond, «Jimmy de Jacques Poulin», les Cahiers François-Xavier Garneau, no 2, 1970, p. $76-78$ (reproduit dans le Lien, janvier 1970, p. 10).

BONSIGNORE, Giacomo, «Jacques Poulin: une conception de l'écriture», Études françaises, vol. XXI, no 3, hiver 1985-1986, p. 19-26.

BOSCO, Monique, «Un menteur infiniment poétique», le Magazine Maclean, mai 1969, p. 70.

BOURNEUF, Roland, «Jacques Poulin, Faites de beaux rêves», Livres et auteurs québécois, 1974, p. 51-53.

BOURQUE, Paul-André, «La fascination de l'enfance, de la tendresse et de la mort chez Jacques Poulin ou la recherche de l'androgyne absolue», Nord, n 2, hiver 1972, p. 74-92.

—, «L'art de communiquer l'incommunicabilité», Québec français, mai 1979, p. 38-39 (reproduit dans Romanciers du Québec, Québec, Québec-français, 1980, p. 155159).

CARRIER, Roch, «Introduction», The Jimmy Trilogy, Toronto, Anansi, 1979, p. 6-7.

-, «Jacques Poulin: Toronto: Montréal», Nord, no 2, 1972, p. 73.

CASSIDY, Jim, «Great Characters in Need of Further Study», Vancouver Sun, 16 avril 1988 , p. D-4.

CHAPUT, Sylvie, «Les Grandes Marées», le Bulletin Pantoute, no 2, juin-juillet-août 1980, p. 24.

CHASSAY, Jean-François, «Un écrivain américain», Spirale, septembre 1984, p. 8.

CHOQUETTE, Sylvie, «L'archétype du temps circulaire chez Emest Hemingway et Jacques Poulin", Etudes littéraires, vol. VIII, no 1, avril 1975, p. 43-55.

CLOUTIER, Rachel, Rodrigue GIGNAC, Vincent NADEAU et Richard PLAMONDON, «Entrevue avec Jacques Poulin», Nord, no 2, hiver 1972, p. 9-29.

C.M.R., «Les Prix de la Presse à Hubert Aquin et à Jacques Poulin», le Soleil, 25 octobre 1974, p. C-5.

COSSETTE, Gilles, «Jimmy», Livres et auteurs québécois, 1969, p. 12-13.

DAVIES, G., «The Jimmy Trilogy: Book Review», The Fiddlehead, no 129, printemps 1981, p. 128-129. 
DÉCARIE, Nicole, «Volkswagen Blues», Mrbius, n²2, été 1984, p. 100-101.

DEMERS, Jeanne, «Le Cour de la baleine bleue de Jacques Poulin», Livres et auteurs québécois, 1970, p. 46-47.

- "Besoin de tendresse, over" ou Jacques Poulin conteur, Etudes françaises, vol. XXI, no 3, hiver 1985-1986, p. 27-35.

DESROSIERS, Jacques, «Hertel pour un cheval», l'Écho du Nord, 17 janvier 1968, p. 38,55 .

DORION, Gilles, «La littérature québécoise contemporaine: 1960-1977 II. Le roman», Études françaises, vol. XIII, nos 3-4, octobre 1977, p. 329-331.

-, «Les Grandes Marées», Québec français, octobre 1978, p. 6.

-, "Jimmy», Dictionnaire des cuvres littéraires du Québec, tome IV, 1960-1969, Montréal, Fides, 1984, p. 469-471.

_, «Volkswagèn Blues», Québec français, octobre 1984, p. 8.

DORION, Gilles et Cécile DUBÉ, «Jacques Poulin», Québec français, mai 1979, p. 3335 (reproduit dans Romanciers du Québec, Québec, Québec-français, 1980, p. 147 154).

DUBE, Cécile, «Piste de lecture», Québec français, mai 1979, p. 36-37.

DUQUETTE, Jean-Pierre, «Les Grandes Marées», Livres et auteurs québécois, 1978, p. 19.

ETHIER-BLAIS, Jean, «Mon cheval pour un royaume de Jacques Poulin», le Devoir, 30 juin 1967, p. 15.

-, «Jimmy», Dictionnaire des auvres littéraires du Québec, tome IV, 1960-1969, Montréal, Fides, 1984, p. 469-471.

- «Le coeur (emprunté) de la baleine bleue», le Devoir, 23 janvier 1971, p. 11.

FALZONI, Franca Marcato, «Storia di una lingua divenuta impotente. Les Grandes Marées di Jacques Poulin», Atti del 60 Convegno internazionale di studi canadesi, Selva di Fasano, Schema editore, 1985, p. 263-281.

FILION, Pierre, «"La marche des mots” propos contacts», Etudes françaises, vol. XXI, no 3, hiver 1985-1986, p. 97-102.

FISCHMAN, Sheila, «Teddy Bear, Translator», The Montreal Star, 1er avril 1978, p. D-3.

FRASER, Graham, «French Explorers Revisited», The Globe and Mail, 18 août 1984, p. E-13.

FRENCH, William, «Quebec Changes: Reviews of Spring Tides and The Legacy», The Globe and Mail, 26 juillet 1986, p. D-15.

-, «Quebec Novel Takes a Journey in a Dream Machine», The Globe and Mail, 14 avril 1988, p. A-20.

GALLAYS, François, «Faites de beaux rêves», Dictionnaire des cuuvres littéraires du Québec, tome V, 1970-1975, Montréal, Fides, 1987, p. 332-333.

GARNEAU, Jacques, «Le Cour de la baleine bleue ou le labyrinthe intérieur», Nord, n० 2, hiver 1972, p. 59-68.

GARNEAU, Jacques, «Le Caur de la baleine bleue», Dictionnaire des auvres littéraires du Québec, tome V, 1970-1975, Montréal, Fides, 1987, p. 161-162.

GAUDET, Gérald, «Adolescente Amérique», le Sabord, no 4, automne 1984, p. 6.

GAUVIN, Lise, «Une voix discrète», le Devoir, 29 avril 1978, p. 33.

-, «Romans», University of Toronto Quarterly, vol. XLVIII, no 4, été 1979, p. 331. 
GAY, Paul, «Jimmy», le Droit, 19 juillet 1969, p. 7.

—, «Le cheval de Jacques Poulin», la Presse, 30 juin 1967, p. 16.

GILBERT, Bernard, «Volkswagen Blues», Inter, no 25, automne 1984, p. 52.

GODIN, Jean-Cléo, «Entre la pierre et l'extase», Nord, n॰ 2, hiver 1972, p. 38-47.

GODBOUT, Jacques, «Les Grandes Marées», l'Actualité, vol. III, no 8, août 1978, p. 55.

GOLDIE, Terry, «Transcontinental», Books in Canada, vol. XVII, no 6, août-septembre 1988, p. 217.

GRADY, Wayne, «The Jimmy Trilogy: Accent on the Soul», Books in Canada, vol. IX, no 1 , janvier 1980 , p. 14-15.

HAMEL, Réginald, John HARE et Paul WYCZYNSKI, Dictionnaire pratique des auteurs québécois, Montréal, Fides, 1976, p. 568-569.

HANDFIELD, Micheline, «Le Cour de la baleine bleue: l'histoire d'une enfance inaccessible», Québec-presse, 17 janvier 1971, p. B-18.

HÉBERT, François, «Le Noir et le Blanc, le Bleu et le Rouge», Études françaises, vol. XI, no 2, mai 1975, p. 117.

—, «Deux maitres livres issus sur l'humour», le Devoir, 12 mai 1984, p. 25.

HÉBERT, Pierre, «De la représentation de l'espace à l'espace de la représentation», Études françaises, vol. XXI, no 3, hiver 1985-1986, p. 37-53.

—. «Romans», University of Toronto Quarterly, vol. LIV, no 4, été 1985, p. 346-347.

HOMEL, D. «Spring Tides; Jacques Poulin», Essays on Canadian Writing, no 36, 1988, p. 87-89.

HUNT, Douglas, «The Jimmy Trilogy», Quill \& Quire, vol. LXVI, n० 4, avril 1980, p. 34.

JANELLE, Claude, «Des enfants perdus dans le grand monde», le Jour, 24 août 1974, p. 12.

—, les Éditions du Jour: une génération d'écrivains, Montréal, Hurtubise/HMH, 1983, 338 p. (Cahiers du Québec, coll. Littérature, no 73).

JARMAN, Marc Anthony, «Volkswagen Blues; Jacques Poulin», Malahat Review, no 83, 1988, p. 187-188.

—, «This Slow Volkswagen Needs Turbocharging», Calgary Herald, 28 août 1988, p. F-5.

LABERGE, Pierre, «Le rejet. À propos du Cour de la baleine bleue», l'Action, 20 février 1971, p. 19.

LABONTÉ, René, «Québec-Californie: la Californie à travers la fiction littéraire québécoise», The French Review, vol. LXII, no 5, avril 1989, p. 803-814.

LACHANCE, Maurice, «Une (ou deux) voix dans l'orchestre», Études françaises, vol. XXI, no 3, hiver 1985-1986, p. 55-65.

LAURIN, Michel, «Le livre du mois: Volkswagen Blues de Jacques Poulin», le Progrès, 30 juillet 1984, p. 15.

—, «Volkswagen Blues», Nos livres, vol. XV, nos 5774-5823, juin-juillet 1984.

LEDGER, Brent, «Poulin's Witty Trek: Quebec's Reach for an American Home», Quill \& Quire, vol. LIV, no 6, juin 1988, p. 26.

LEITH, Linda, «The Walls of Old Quebec», Canadian Forum, vol. LX, no 700, juinjuillet 1980, p. 34-35.

LEROUX, Odette, «Mon cheval pour un royaume de Jacques Poulin», Livres et auteurs québécois, 1967, p. 58. 
LEVASSEUR, Jean, «L'ambiguitté temporelle dans Jimmy de Jacques Poulin», l'Espacetemps dans la littérature, les Cahiers de l'APFUCC, série III, no 2, 1989, p. 39-52.

LOCKQUELL, Clément, «Deux peintures sur la stérilité», le Soleil, 8 juillet 1967, p. 25.

MAILHOT, Laurent, «Le voyage total», Etudes françaises, vol. XXI, n 3, hiver 19851986, p. 3-5.

- «Bibliothèques imaginaires: le livre dans quelques romans québécois», Etudes françaises, vol. XXI, no 3, hiver 1985-1986, p. 81-92.

MAJOR, André, «Mon cheval pour un royaume», le Devoir, 8 juin 1967, p. 6.

—, «Entrevues. Un poète et un romancier», le Devoir, 1 1َr mars 1969, p. 15.

—, «limmy», le Devoir, 15 mars 1969, p. 15.

-, «Jacques Poulin et Gilles Archambault. A la recherche de la bonne tendresse», le Devoir, 22 mars 1969, p. 15 (reproduit sous le titre «Jimmy de Jacques Poulin», Québec 1969, vol. VI, mai 1969, p. 124-127).

MARCOTTE, Gilles, «Comptes rendus. Jacques Poulin. Jimmy», Etudes françaises, vol. V, no2, mai 1969, p. 236-237.

—, «Lisez Jacques Poulin, faites de beaux rêves», le Devoir, 12 mai 1979, p. 23.

—, «L'ambitieuse Volkswagen», l'Actualité, vol. IX, n 8, août 1984, p. 79.

_. «Histoires de zouaves», Études françaises, vol. XXI, no 3, hiver 1985-1986, p. 7-17. Reproduit dans Littérature et circonstances. Essais, Montréal, 1'Hexagone, 1989.

—, «Poulin chez les hommes (Jimmy)», les Bonnes Rencontres: chroniques littéraires, Montréal, HMH, 1971, p. 205-207.

MARTEL, Réginald, «C'est d'enfance qu'il s'agit», la Presse, 8 mars 1969, p. 23.

—, «Le voyage au pôle noir», la Presse, 23 janvier 1971, p. D-3.

—. «alut, Frère Jacques!», Nord, no 2, hiver 1972, p. 69-70.

--, «Du bon usage de la tendresse», la Presse, 22 juin 1974, p. C-3.

-, «Un très beau livre qui reste ouvert», la Presse, 13 mai 1978, p. D-3.

—, «L'Amérique entre rêve et violence», la Presse, 12 mai 1984, p. D-3.

MELANÇON, Benoît, «Roman américain, Jacques Poulin: Volkswagen Blues», Canadian Literature, no 103, hiver 1984, p. 111-113.

MERIVALE, Patricia, «The Jimmy Trilogy», Canadian Literature, no 88, printemps 1981, p. 128-129.

MEZEI, Kathy, «The Jimmy Trilogy», Queen's Quarterly, vol. LXXXVII, no 3, automne 1980 , p. 518-519.

MICHAUD, Ginette, «Récits postmodernes», Etudes françaises, vol. XXI, no 3, hiver 1985-1986, p. 67-88.

MICHON, Jacques, «Heureux qui comme Ulysse», Voix \& images, vol. XI, no 1 , automne 1985, p. 135-139.

MILOT, Louise, «Quand les beaux rêves tournent au blues: le Volkswagen Blues de Jacques Poulin», Lettres québécoises, no 35, automne 1984, p. 15-17.

MONTESSUT, Carmen, «Les Grandes Marées», le Journal de Montréal, 29 novembre 1981, p. 62.

MORENCY, Pierre, «Le plus grand menteur de la ville de Québec ou lettres à Jacques Poulin», Nord, n² 2, 1972, p. 48-58.

NACHTSHEIM, M.H., «Spring Tides», Choice, vol. XXIV, février 1987, p. 889.

NOLIN, Jacques, «Les Grandes Marées», Nos livres, vol. IX, no 250, juin-juillet 1978. 
O'CONNOR, John J., «Translations», University of Toronto Quarterly, vol. XLIX, no 4, été 1980, p. 384-385.

OUELLETTE, Gabriel-Pierre, «Jacques Poulin: les Grandes Marées», Livres et auteurs québécois, 1978, p. 71-74.

OUELLETTE-MICHALSKA, Madeleine, «Les Grandes Marées», Châtelaine, vol. XIX, no 8, août 1978, p. 16.

PAGÉ, Raymond, «Les Grandes Marées», The Chelsea Journal, vol. V, n० 5, septembreoctobre 1979, p. 232-233.

PELLETIER, Jacques, «Faites de beaux rêves», Livres et auteurs québécois, 1974, p. 17.

PHILPOTTS, Jane, «Quiet Reading of Jacques Poulin's Trilogy Leaves Pleasant Taste», Calgary Herald, 29 décembre 1979, p. C-10.

POISSON, Roch, «Bloc-notes», Photo-Journal, 21-28 juin 1967, p. 68.

PONTAUT, Alain, «Mon étalon pour une calèche», la Presse, 1er juillet 1967, p. 21.

—, «Un blanc provisoire pour le creux de l'été», la Presse, 19 août 1967, p. 21.

POULIN, Gabrielle, «Le Cour de la baleine bleue de Jacques Poulin, un roman piégé», Relations, mai 1972, p. 154-155 (reproduit dans Romans du pays 1968-1979, Montréal, Bellarmin, 1980, p. 263-270).

- «Les Grandes Marées de Jacques Poulin ou la genèse en bandes dessinées», Relations, no 437, mai 1978, p. 154-156.

POULIN, Gabrielle et René DIONNE, «Romans, récits, nouvelles, contes», University of Toronto Quarterly, vol. XLIV, n 4, été 1975, p. 321.

QUIGLEY, Theresa, «A Crowded Eden», The Fiddlehead, no 153, automne 1987, p. $102-103$.

RAFELMAN, Rachel, «Spring Tides», Quill \& Quire, vol. LII, octobre 1986, p. 46.

RASPORICH, Beverly, «The Jimmy Trilogy», Canadian Book Review Annual, 1979, p. 103.

RENAUD, André, «Les Grandes Marées», Voix \& images, vol. V, n 2, automne 1979, p. 193-195.

RICARD, François, «Jacques Poulin: Charlie Brown dans la Bible», Liberté, vol. XX, no 3, mai-juin 1978, p. 85-88.

—, «Jacques Poulin; de la douceur à la mort», Liberté, vol. XVI, nos 5-6, septembredécembre 1974, p. 97-105.

RIOUX, Gilles, «Enfin un roman contemporain», Sept-Jours, 1er juillet 1967, p. 48.

ROBIDOUX, Réjean, «Romans, récits, nouvelles et contes», University of Toronto Quarterly, vol. XL, no 4, été 1971, p. 424.

ROUX, Paul, «Jimmy: un grand roman sur l'enfance», le Soleil, 15 mars 1969, p. 32.

ROY, Michel et Jean GAUTHIER, «Conversation avec Jacques Poulin», la Revue Pantoute, no 3, septembre-octobre-novembre 1980, p. 22-23.

ROYER, Jean, «Jacques Poulin, romancier artisan», le Devoir, 29 avril 1978, p. 33.

RUDEL-TESSIER, Danièle, «Volkswagen Blues», Châtelaine, vol. XXV, no 9 , septembre 1984, p. 43.

SAINT-ONGE, Paule, «Mon cheval pour un royaume», Châtelaine, vol. VIII, no 9 , septembre 1967, p. 28.

_-, «Des voix qu'on aime entendre», Châtelaine, vol. X, n॰ 5, mai 1969, p. 42.

_-, «Explorer l'enfance toute proche ou les pays lointains», Châtelaine, vol. XII, n 4, avril 1971, p. 26. 
SÉVIGNY, Marc, «Cinéma québécois: les écrivains à la rescousse», Nuit blanche, nº 10 , automne 1983, p. 48-51 (sur le film les Grandes Marées).

SIMON, Sherry, «Quebec Writer, Metis Girl, Go to Look for America», The Montreal Gazette, 21 mai 1988, p. L-12.

SOULIÉ, Jean-Paul, «Jacques Poulin après Volkswagen Blues: d'abord vivre une histoire d'amoun, la Presse, 7 juillet 1984, p. B-3.

SPETTIGUE, D.O., «Spring Tides; Jacques Poulin; Book review», Queen's Quarterly, vol. XCIV, no 2, été 1987, p. 366-375.

TASCHEREAU, Yves, «Volkswagen Blues», le Livre d'ici, vol. IX, no 9, mai 1984, p. 23. THÉBERGE, Jean-Yves, «L'excellent Jimmy», le Canada français, 28 mai 1969, p. 44.

- «Une histoire de cœur, mais du cour d'une autre», le Canada français, 17 février 1971, p. 26.

TREMBLAY, Régis, «Poulin: la fraternité est la nouvelle frontière», le Soleil, 7 juillet 1984, p. D-1.

- «Déshabiller la phrase de ses adjectifs», le Soleil, 7 juillet 1984, p. D-2.

TREMBLAY, Robert, «Jacques Poulin devant le dur métier d'écrire», le Soleil, 2 novembre 1974, p. D-6.

_-, «Un roman qui a la politesse du désespoir», le Soleil, 2 novembre 1974, p. D-6.

TREMBLAY, Yolaine, «Rencontre avec Jacques Poulin», Azimuth, décembre 1976, [n.p.].

_, «Volkswagen Blues par Jacques Poulin», le Livre d' ici, mai 1984, p. 23.

URBAS, Jeannette, «A Teddy Bear's Tale», Canadian Forum, vol. LXVI, no 765, janvier 1987, p. 39.

URQUART, J., «Spring Tides by Jacques Poulin: Book Review», Canadian Literature, no 116, printemps 1988, p. 129-131.

VALIQUETTE, Bernard, «Mon cheval pour un royaume», Échos-Vedettes, 19 août 1967, p. 21.

VACHON, G.-André, «Le roman: une littérature qui se louisianise», Etudes françaises, no 4, vol. V, novembre 1971, p. 412-414.

VASSEUR, François et Michelle ROY, «Voyage à travers l'Amérique», Nuit blanche, no 14, juin-août 1984,p. 50-52.

WEISS, Jonathan M., «Une lecture américaine de Volkswagen Blues», Études françaises, vol. XXI, no 3, hiver 1985-1986, p. 89-102.

WILLIAMSON, David, «Gentle Fable of a Lost Eden: Review of Spring Tides», Winnipeg Free Press, 9 août 1986, p. 50.

-, «Quebec Novelist Offers Lesson in Joyous, Gentle Writing», Winnipeg Free Press, 30 avril 1988, p. 7-A. 